\title{
Membranes of Semicrystalline Aliphatic Polyamide Nylon 4,6: Formation by Diffusion-Induced Phase Separation
}

\author{
A. M. W. BULTE, * B. FOLKERS, M. H. V. MULDER, and C. A. SMOLDERS \\ University of Twente, Faculty of Chemical Technology, P.O. Box 217, 7500 AE Enschede, The Netherlands
}

\begin{abstract}
SYNOPSIS
The preparation of membranes of nylon 4,6 by diffusion-induced phase separation (DIPS) using formic acid as a solvent and water as a nonsolvent was studied. Nylon 4,6 is a semicrystalline polymer; phase separation from a solution can occur by solid-liquid (s-l) demixing as well as by liquid-liquid (1-1) demixing. Upon quenching films of solutions with low polymer concentration $(<17 \mathrm{wt} \%)$ in a nonsolvent bath containing water, the morphology of the membranes show a foam-like structure typical for l-l demixing. When phase separation is induced by water vapor a transition in structure occurs from the cellular type to a morphology typical for $\mathrm{s}-1$ phase separated films. At higher polymer concentrations membranes exhibit structures consisting of spheres or smaller crystal-like units resulting from an s-l phase separation process. The addition of $2 \mathrm{wt} \%$ or more of water to polymer solutions with low concentration (up to $15 \mathrm{wt} \%$ ) resulted in $\mathrm{s}-1$ demixing as well. In a DIPS process $\mathrm{s}-\mathrm{l}$ demixing is kinetically competitive with $1-1$ demixing if nuclei are already present in the starting solutions (heterogeneous nucleation), or if a relatively long time is available for crystal nuclei to be formed. The morphology resulting from $\mathrm{s}-\mathrm{l}$ demixing is a result of spherulitic crystallization. A certain concentration of nuclei or of precursor particles already present results in a small nucleation density during precipitation and thus large spherulites can be grown; at higher polymer and/or water concentrations the nucleation density increases resulting in an axialitic morphology of the membranes. (C) 1993 John Wiley \& Sons, Inc.
\end{abstract}

\section{INTRODUCTION}

The use of aliphatic polyamides, such as nylon 6 , nylon 6,6, and nylon 11 , as a membrane material is of growing importance: most applications are found in microfiltration ${ }^{1}$ for which preparation of nylon membranes has been described in patents. It is expected that aliphatic polyamides will be used in a much broader field because of their favorable mechanical properties and chemical stability.

Membranes can be prepared by various techniques one of which is immersion precipitation, a widely used technique. ${ }^{2}$ A polymer solution is cast

\footnotetext{
* To whom all correspondence should be addressed.
} Journal of Applied Polymer Science, Vol. 50, 13-26 (1993) (C) 1993 John Wiley \& Sons, Inc.

CCC 0021-8995/93/010013-14 as a thin film and subsequently immersed in a nonsolvent bath, thereby inducing diffusion-controlled phase separation (DIPS). The liquid-liquid (1-1) demixing process that usually takes place for amorphous and low-crystalline polymers has been studied extensively in our laboratory. ${ }^{3-5}$ Solid-liquid ( $\mathrm{s}-1$ ) demixing in membrane formation has also been described ${ }^{6,7}$ Lloyd et al. studied the thermally induced phase separation process (TIPS) of isotactic polypropylene and polyethylene solutions. In recent work an extensive study on the different possibilities of demixing processes of a binary system was presented..$^{8-12}$

Our objective is to investigate the process of $\mathrm{s}-1$ demixing behavior of films cast from a nylon 4,6 solution in a diffusion-induced demixing process in a ternary system.

Crystallization phenomena in (quasi) ternary 
mixtures for membrane formation have been studied in our laboratory ${ }^{13}$ for the system cellulose acetate, dioxane, and water; by van Emmerik and Smolders, ${ }^{14}$ Koenhen and Smolders, ${ }^{15}$ and Wijmans et al. ${ }^{16}$ for polyphenylene oxide (PPO); and by Reuvers ${ }^{17}$ using both PPO and cellulose acetate as a membrane material. The studies on crystallization in membrane formation started with a systematic study on the behavior of PPO in different solutions. ${ }^{15}$ Wijmans et al. ${ }^{16}$ studied the ternary system of a solution of PPO in trichloroethane (TCE) with methanol or octanol as nonsolvents. Burghardt et al. ${ }^{18}$ did the same for methanol, ethanol and octanol; in addition a phase diagram was calculated for ethanol as nonsolvent. The phase separation process responsible for membrane formation is $1-1$ demixing in this case. PPO membranes generally do not show any melting peak in a differential scanning calorimeter (DSC) measurement, indicating that the membrane has a low crystallinity. Both Reuvers ${ }^{17}$ and Burghardt ${ }^{18}$ found that in some cases crystallization is the thermodynamically preferred phase separation process. The kinetically faster $1-1$ demixing processes for PPO, however, are favorable compared to the relatively slow crystallization process, if fast quenching conditions are applied for membrane preparation.

The work described on DIPS so far indicates that crystallization can influence the membrane structure but the nature of the demixing process is still unknown. Agglomerates formed during the demixing process are called aggregates. ${ }^{19}$ Aggregate formation of lamellar type can be grown from dilute solutions and more complex morphologies, for example, dendrites and spherulites are formed from solutions of medium concentrations upon decreasing the thermodynamic quality of a polymer solution by loss of solvent, by lowering the temperature, or by introducing a nonsolvent.

From the literature it became clear that aliphatic polyamide membranes were formed according to an s-l demixing process. ${ }^{20-21}$ In our work nylon 4,6 was chosen as a material for membrane formation because of its promising properties for membrane applications in terms of chemical stability.

The aim of the present work is to study:

1. the phenomenological effects of various membrane formation parameters and

2. the features of this polymer as a model system for a process in which both $\mathrm{s}-\mathrm{l}$ and $1-1 \mathrm{de}$ mixing can occur during immersion precipitation.

\section{THEORY}

\section{Membrane Formation in Ternary Systems}

Membrane morphologies obtained by immersion precipitation are usually a result of $\mathrm{l}-\mathrm{l}$ demixing of the ternary system polymer, solvent, and nonsolvent. A dissolved polymer film is cast onto a support. This is then immersed into a bath containing nonsolvent. Exchange of solvent and nonsolvent leads to instability of the polymer solution. Pores in the final membrane are a result of nuclei that are grown from the polymer lean phase. The morphology of such a membrane can be visualized as a cellular or foam-like structure.

\section{Semicrystalline Polymers}

A semicrystalline polymer in a diluent can decrease its free enthalpy by separation of the homogeneous solution into a phase that consists of a pure crystalline polymer and a remaining phase that is smaller in polymer concentration ( $\mathrm{s}-1$ demixing). In such a membrane pores are the voids between the crystalline structures.

The thermodynamic background of s-1 demixing is shown for the binary case with one crystallizable polymer and one diluent (Fig. 1). If no $1-1$ demixing takes place, the free enthalpy of mixing $\left(G_{M}\right)$ curve is concave and strongly asymmetric for a system with one polymer.

A polymer solution with $\phi_{A}<\phi_{P}<1$, in which $\phi_{P}$ represents the volume fraction of the polymer, can lower its free enthalpy of mixing by phase separation into a pure crystalline state $\left(\phi_{P}=1\right)$ and a more diluted phase $\left(\phi_{P}=\phi_{A}\right)$ at a temperature $T_{A}$. At equilibrium the chemical potentials of the polymer repeating unit in the liquid phase and in the crystalline polymer must be equal. $T_{A}$ is called the melting temperature, $T_{m}$, of a solution with composition $\phi_{A}$. At temperatures larger than $T_{A}$ the chemical potential of the crystalline state, $\mu_{C}$, shifts to larger values. The free enthalpy of the mixing curve continues to have a similar shape, but at higher temperatures the equilibrium melting temperature shifts to compositions with larger polymer concentrations [Fig. 1(b)].

The complete melting point depression curve can be described according to Flory ${ }^{22}$ by:

$$
\frac{1}{T_{m}}-\frac{1}{T_{m}^{o}}=\left(\frac{R}{\Delta H_{u}}\right)\left(\frac{V_{u}}{V_{d}}\right)\left(\phi_{d}-\chi_{p d} \phi_{d}^{2}\right)
$$


a)

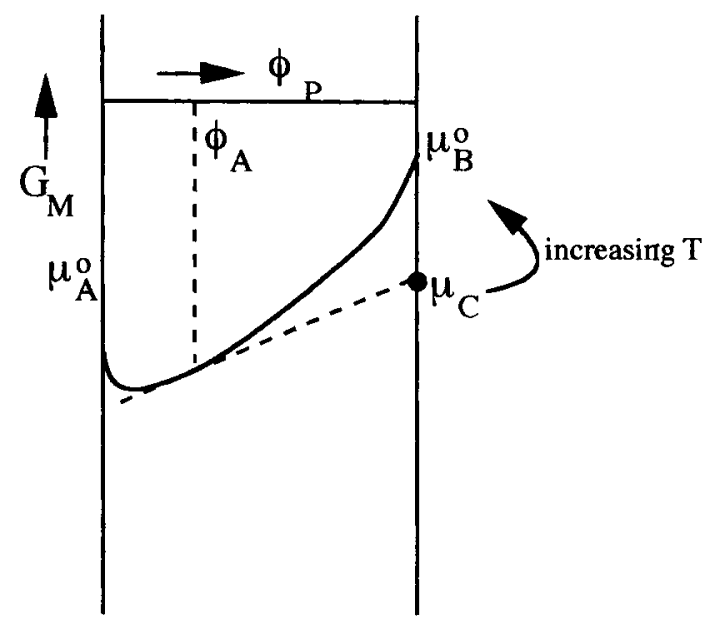

b)

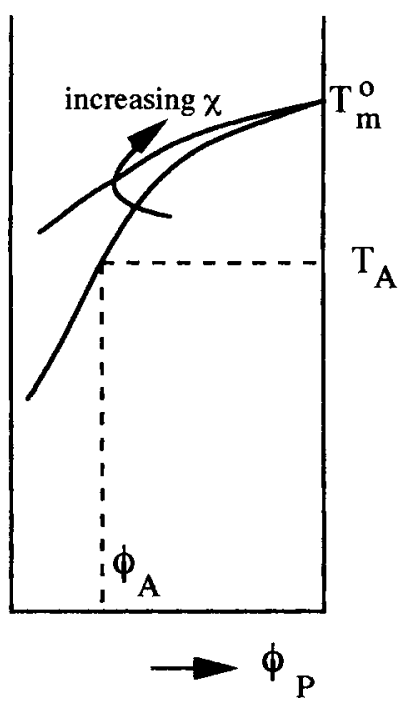

Figure 1 (a) Free molar enthalpy of mixing $\left(G_{M}\right)$ at a temperature $T_{A}$ as a function of the volume fraction of the polymer $\left(\phi_{P}\right)$ for a binary system with one diluent and one polymer. (b) A binary phase diagram constructed from a $G_{M}-\phi_{P}$ diagram [ see (a)] according to the principle of equal tangent lines.

in which $T_{m}$ and $T_{m}^{o}$ are the melting temperatures of the diluted polymer and of the pure polymer, respectively; $\Delta H_{u}$ the heat of fusion per mole of repeating units of polymer; $V_{d}$ and $V_{u}$ the molar volume of the diluent and the repeating unit of polymer, respectively; $\phi_{d}$ is the volume fraction of the diluent; and $\chi_{p d}$ the interaction parameter polymer diluent.

The thermodynamic relation of chemical potentials of the crystalline phase and the diluent phase in a ternary system is more complicated. Altena et al. ${ }^{23}$ derived an expression for the melting point depression of the polymer (3) in a ternary system containing solvent (2) as well as nonsolvent (1) using concentration independent Flory Huggins parameters, $\chi_{12}, \chi_{13}$, and $\chi_{23}$. Burghardt et al..$^{18}$ derived a relation for concentration dependent interaction parameters. It is very difficult, however, to obtain all the concentration dependent parameters for a ternary system; therefore we tried to understand the behavior of the melting point depression for a ternary system in a more intuitive manner. It is expected that the lower the equilibrium melting temperature, the better the solvent for the polymer. This was discussed by $\mathrm{Paul}^{24}$ using the approach of solubility parameters. ${ }^{25}$ Figure 1 (b) shows how a melting point depression curve may change with an increasing interaction parameter, $\chi_{p d}$. When the diluent phase contains more nonsolvent, the diluent is a poorer solvent for the polymer. With a constant polymer concentration the equilibrium melting temperature increases when the nonsolvent content is larger.

For a ternary system a three-dimensional plot can be constructed as shown in Figure 2. The curve in the polymer-solvent plane (curve $A$ in the left-hand plane of the triangle diagram) represents the melting point depression curve for the polymer-solvent system. Curve $B$ represents polymer solutions in which a certain constant ratio of solvent and nonsolvent is present. The cross section of the different equilibrium lines with the temperature plane at $30^{\circ} \mathrm{C}$ gives the equilibrium (isothermal) crystallization line in the ternary phase diagram at $30^{\circ} \mathrm{C}$. Since the curves for larger nonsolvent concentrations and identical polymer concentration lie at higher $T$ values than the polymer-solvent line, the isothermal crystallization line shifts to lower polymer concentration for larger nonsolvent concentrations.

An equilibrium crystallization line in the phase diagram represents s-l demixing. For membrane forming systems also an l-l demixing gap is present, which results in a l-l demixing process. Figure 3 gives a possible phase diagram for a ternary system in which the polymer can crystallize. Point A represents, as stated, the crystallization of polymer $(\mathrm{P})$ in solvent $(\mathrm{S})$. The curve $A-B$ is the crystallization 


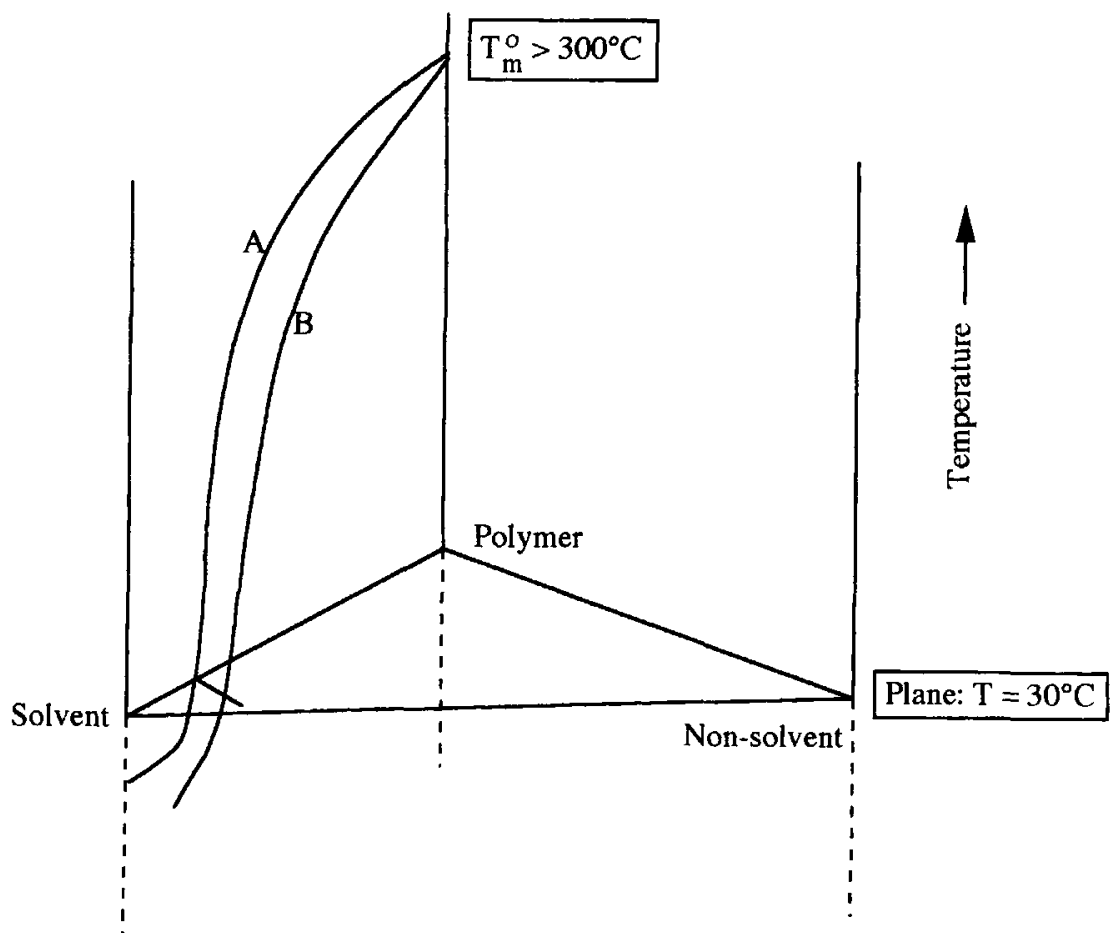

Figure 2 The ternary phase diagram with the equilibrium melting curve as a function of composition and temperature. The solvent polymer plane gives the binary situation of melting-point depression. The cross section at $T=30^{\circ} \mathrm{C}$ gives the isothermal phase diagram with the solid-liquid demixing border at equilibrium conditions.

curve. Any composition inside the area A-B-P can crystallize into pure polymer $\mathrm{P}$ and a composition on curve $A-B$. Curve $B-D-C$ is the $1-1$ demixing curve: the binodal. Compositions inside the area BC-D exhibit phase separation into two liquid phases, connected by a tie-line. Point $\mathrm{D}$ is the critical point where the two equilibrium compositions coincide.

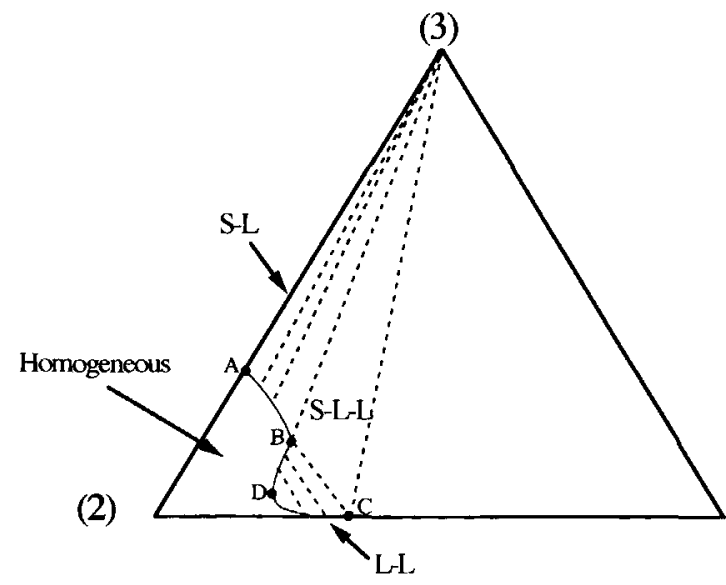

Figure 3 An isothermal ternary phase diagram for a semicrystalline polymer $(P$, index: 3$)$, a solvent $(S$, index: 2 ), and a nonsolvent (NS, index: 1 ).
Area B-C-P is the area in which three phases are in equilibrium: the compositions indicated with $\mathrm{B}$, $\mathrm{C}$, and $\mathrm{P}$. In area $\mathrm{P}-\mathrm{C}-\mathrm{NS}$, phase separation into a solid phase $(\mathrm{P})$ and a liquid phase with a composition on the line $\mathrm{C}-\mathrm{NS}$ will occur.

It is important to notice that a phase diagram represents equilibrium thermodynamics; kinetic effects are not included. During membrane formation, diffusion processes of nonsolvent and solvent continue; equilibrium is never achieved. At a certain position in the film a composition may thermodynamically be meta-stable, but the time scale for demixing can be too short for the demixing process to take place. The crystallization process especially is expected to require a time interval in which polymer chains are allowed to migrate to a growing crystal and to exhibit a surface reaction on the crystal. In addition to this consideration a certain degree of supersaturation is necessary.

\section{EXPERIMENTAL}

Nylon 4,6 (Stanyl KS 400, kindly supplied by DSM), was dissolved at $30^{\circ} \mathrm{C}$ in formic acid (Merck, 
analytical grade $98 \%$ ) in a concentration range of 10-32 wt \%. The polymer was dried in vacuo for at least $24 \mathrm{~h}$ before use; formic acid was used without further purification. Polymer solutions were cast on a glass plate with a thickness of $0.3 \mathrm{~mm}$. The glass plate with the cast polymer solution was immediately immersed in a water bath. After sufficient coagulation time the membranes were removed from the glass plate, rolled in filter paper, and washed with demineralized water for at least $24 \mathrm{~h}$.

The morphology of the membranes was examined by scanning electron microscopy (Jeol JSM T $220 \mathrm{~A}$ ). The samples were wetted in ethanol, cryogenically broken, and dried in vacuo for at least 6 h. A thin layer of gold was deposited on top of the sample with a Balzer Union SCD 040 sputtering apparatus (sputtering conditions: $25 \mathrm{~mA}$ for $2 \mathrm{~min}$ ).

As a first characterization method, the pure water flux, using ultrafiltrated water, was measured with an Amicon ${ }^{\mathrm{TM}}$ set-up at an applied pressure difference of 2 bar. Pore size analysis was performed on a Coulter ${ }^{\circledR}$ Porometer II (ASTM F316-80, 1980; BS 6410: 1984 ) with Coulter ${ }^{\circledR}$ Porofil as a wetting liquid. The sample holder diameter was $25 \mathrm{~mm}$.

Differential scanning calorimetry (Perkin Elmer DSC-4) was used to investigate the crystalline nature of the polymeric membranes.

\section{RESULTS}

The effect of enlarging the polymer concentration from 10 to $32 \mathrm{wt} \%$ on the ultimate membrane morphology can be clearly observed by electron microscopy (Fig. 4). At lower polymer concentrations ( $<17 \mathrm{wt} \%$ ) l-l demixing takes place, and the membranes show a cellular morphology. At concentrations between 20 and 30 wt \% s-1 demixing occurs resulting in a structure formed of spheres. Growth of a nucleus takes place from the polymer-rich phase that consists of ordered crystalline material, surrounded by a highly concentrated phase of amorphous polymer molecules. Apparently the underlying process is spherulitic crystallization. Membranes prepared from a polymer solution with a concentration larger than $30 \mathrm{wt} \%$ show axialitic crystalline units, which are intermediate stages in the development of a spherulite. The growth of crystalline units to a complete spherulite in this case is inhibited.

Using a polymer concentration of 20 wt \% with an increasing amount of water in the initial solution a similar effect can be observed. The membrane is the result of $s-1$ demixing with a spherulitic struc- ture. The spherulite size has decreased from 10 to 2 $\mu \mathrm{m}$ when $5 \mathrm{wt} \%$ of water (nonsolvent) is added to the initial solution. Upon increasing the water concentration in the starting solution the structure changes to a finer morphology. The membrane with $10 \mathrm{wt} \%$ of water in the original solution shows an axialitic structure. Such an effect is also shown in Figure 5 starting with a 15 wt \% polymer solution. The final structure here ( $15 \mathrm{wt} \% \mathrm{P}, 20 \mathrm{wt} \%$ water ) is better described as a dendritic or leafy structure. Table I summarizes the effect of polymer and water concentration on morphology of the membranes.

The performance of the membranes is shown in Table II. Typical microfiltration membranes were synthesized: $0.1 \mu \mathrm{m}$ membranes for the use in, for example, the semiconductor industry; $0.2 \mu \mathrm{m}$ for an absolute sterilizing grade; and $0.45-0.65 \mu \mathrm{m}$ for the use in the beverage industry. Pure water fluxes and air permeability give an indication about the performance of the membranes. In membranes that result from a l-1 demixed morphology, the pores originate from the growth of nuclei of the polymer lean phase; pores in a s-l demixed type of membrane are the voids between the semicrystalline units. It is obvious that a more interconnected pore structure can be obtained with membranes that result from s-1 demixing. Membranes that are prepared via an l-1 demixing often show isolated pores. Very often special precaution must be taken to improve the connectivity of pores in order to avoid too high resistance toward permeation of the membrane. ${ }^{26}$

The larger these solid particles are, the larger the voids, resulting in a larger effective pore size in the membrane. Indeed the pore size gives a larger value for a membrane with a larger crystallite size than for membranes with a finer morphology (see Figs. 4 and 5 in combination with Table II). Skinless membrane structures were easily prepared with nylon 4,6, while membrane manufacturers of nylon 6 and nylon 6,6 membranes had to use rather high solvent concentrations in the coagulation bath ${ }^{27}$ or had to induce phase separation by water vapor ${ }^{28}$ in order to avoid thick skin layers in the membranes.

The DSC thermograms of the membranes can be compared with the material as it was received (Fig. 6 ). The polymer shows a large endothermic peak at $297^{\circ} \mathrm{C}$; the membranes exhibit smaller but very distinct melting endothermic peaks at somewhat lower temperatures. The membranes prepared are clearly semicrystalline in nature. The heat of fusion for the membranes is somewhat less than for the polymer as it was received, indicating that the degree of crystallization is somewhat lower for the membranes.

The morphology of the membranes is finer when 

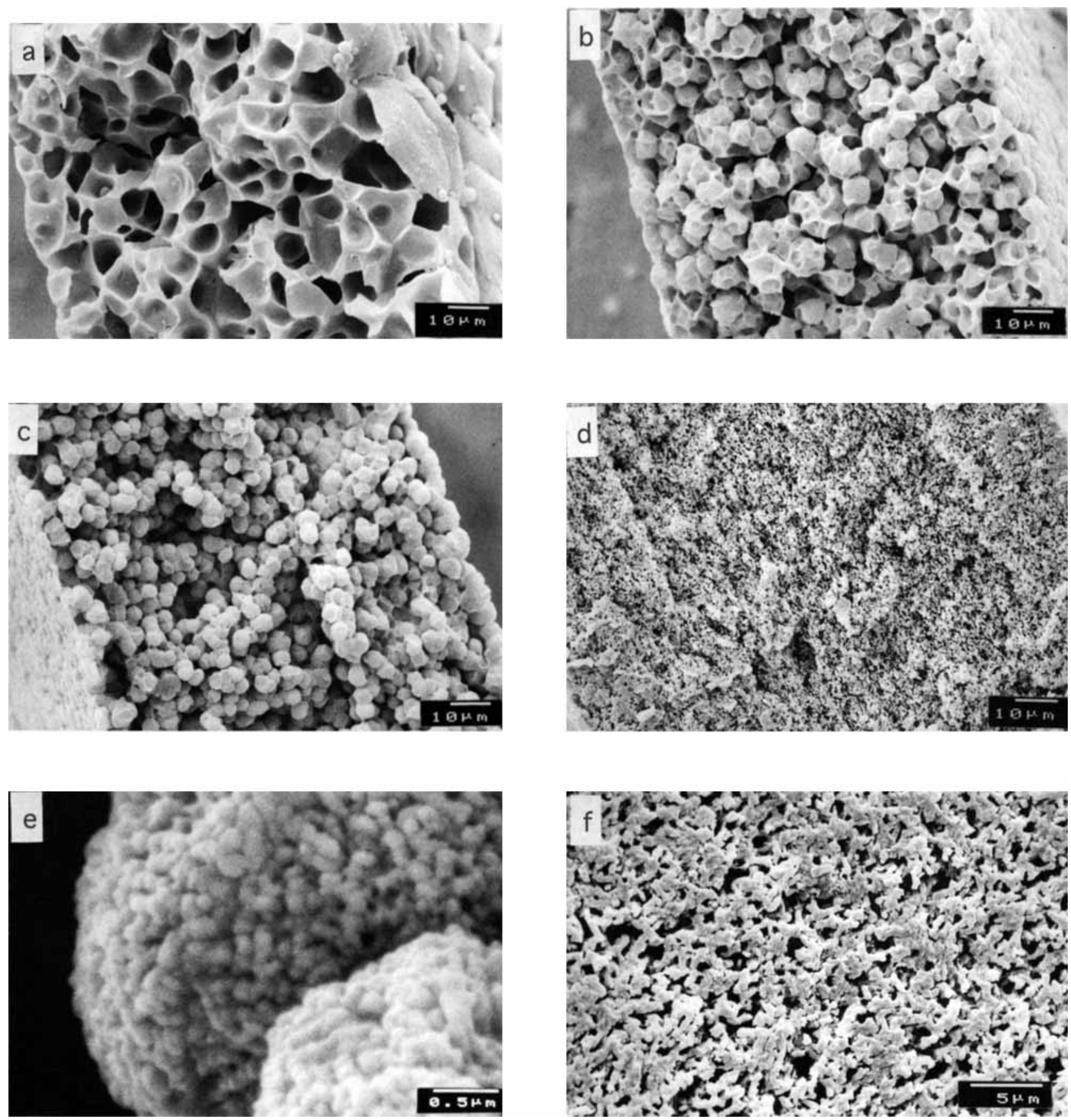

Figure 4 (A) Morphology of nylon 4,6 membranes observed by SEM starting with different polymer concentrations; the polymer concentration (wt \%) is for (a) $15 \%$; (b) $20 \%$; (c) $25 \%$; (d) $32 \%$; (e) and (f) are larger magnifications of photos (c) and (d), respectively.

the polymer concentration or when the water concentration at the same polymer concentration has increased. In order to quantify this behavior a nucleation density is defined as the total number of entities in the membrane structure per unit area of a cross section. Nucleation density is the result of nucleation rate and nucleus growth. Assuming that one unit (a spherulite or axialite for $\mathrm{s}-\mathrm{l}$ demixing or a cell for l-l demixing) has grown from one nucleus, one can count the number of units per area 

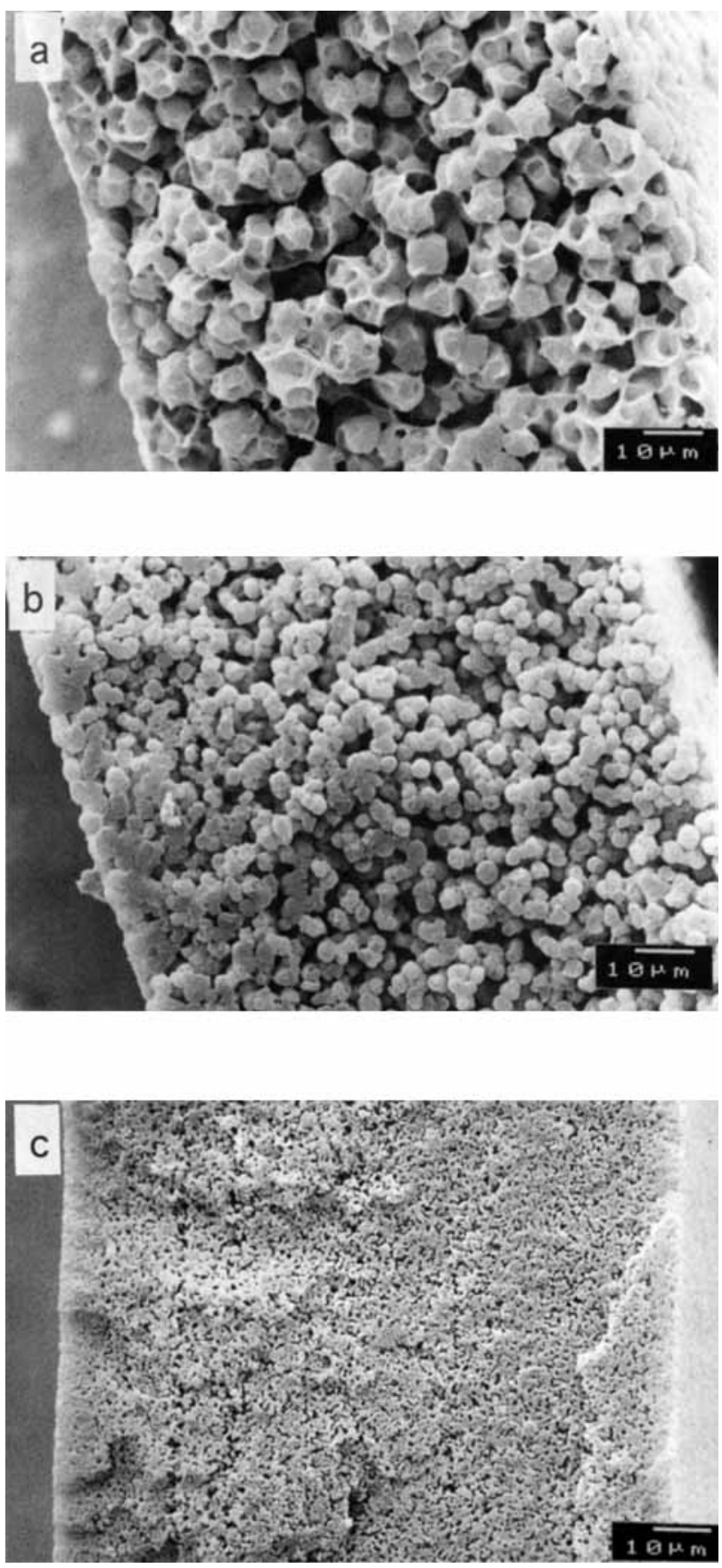

Figure 4 (B) Morphology of nylon 4,6 membranes observed by SEM with different water concentrations (wt \%) in the polymer solution; the polymer concentration of the initial solution is $20 \mathrm{wt} \%$. The water concentration is for (a) $0 \%$; (b) $5 \%$; and (c) $10 \%$.

on a cross section of an SEM picture. Though the structure visualized with SEM is that of a final dry state and membrane formation takes place in the liquid state, nucleation density as it is described above can be compared when the polymer and/or water concentration is varied. Nuclei that have disappeared because of Oswald ripening and/or coalescence are not taken into account.

In Figure 7 the nucleation density is plotted as a function of the ternary solution from which the 

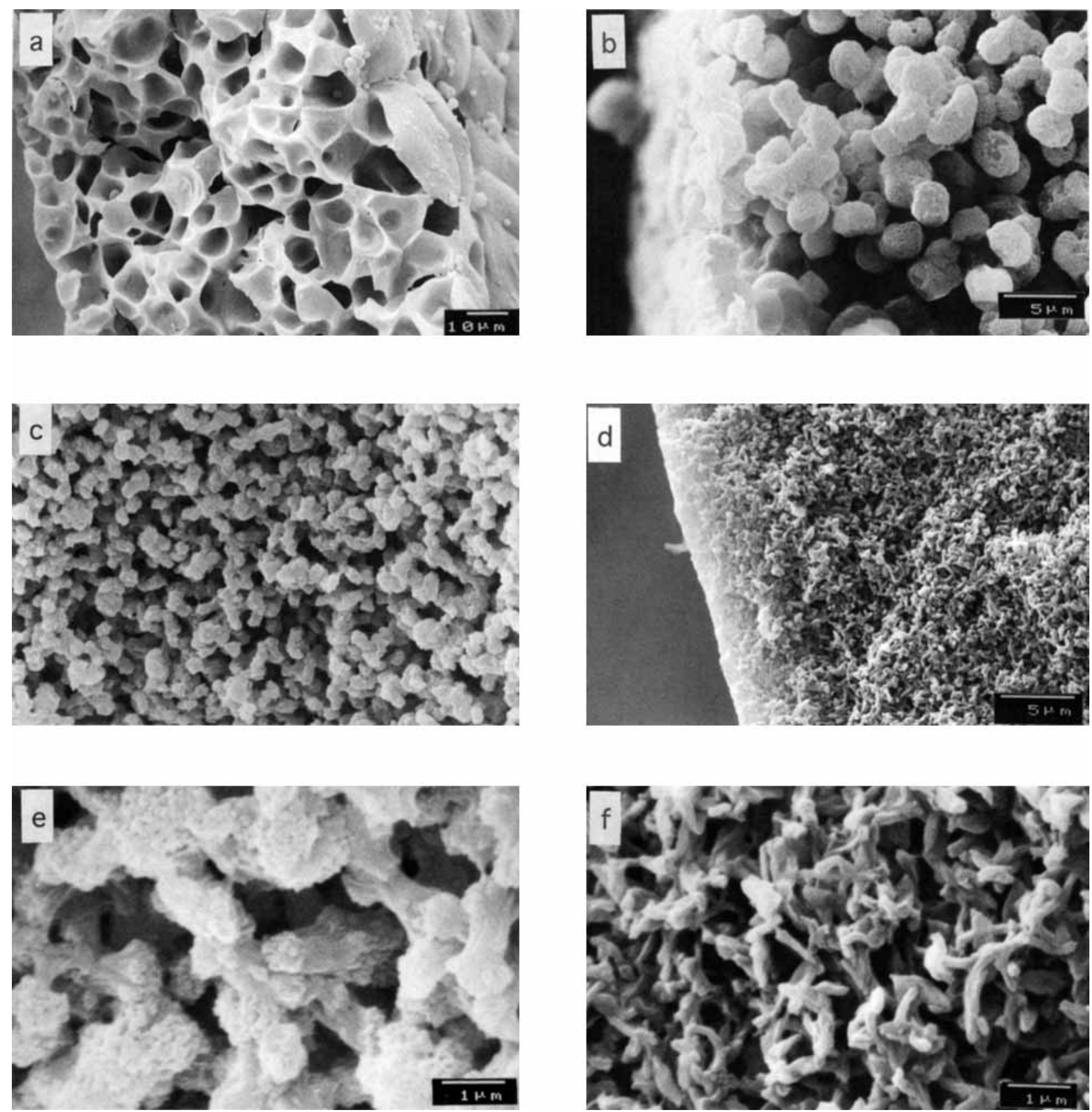

Figure 5 Morphology of nylon 4,6 membranes observed by SEM with 15 wt \% of polymer and different water concentration (wt \%) in the polymer solution (a) $0 \%$; (b) $10 \%$; (c) 15\%; (d) $20 \%$; (e) and (f) are larger magnifications of photos (c) and (d), respectively.

membranes were cast. The dots give the nucleation density for a finer $1-1$ demixing structure; the unit $\mathrm{N}$ then gives the number of cells; the crosses represent the s-l type of demixing. Starting from a low polymer concentration the nucleation density increases nonlinearly with an increasing polymer concentration. The same trend is found for an increasing water content in the polymer solution at a constant polymer concentration.

\section{DISCUSSION}

\section{Spherulite Formation}

As illustrated in Figure 4, some membranes showed a spherulitic structure with spherulites that differ in size from 10 to $2 \mu \mathrm{m}$ for membranes with an increasing polymer and/or water concentration in the starting solution. The schematic development of 
Table I Spectrum of Morphology of Membranes Obtained by Varying Polymer and Water Concentration in Original Polymer Solution

\begin{tabular}{|c|c|c|c|c|c|c|c|c|}
\hline \multirow{2}{*}{$\begin{array}{c}\text { Water } \\
\text { Concentration } \\
(\text { wt } \%)\end{array}$} & \multicolumn{8}{|c|}{ Polymer Concentration (wt \%) } \\
\hline & 10 & 12 & 15 & 17 & 20 & 25 & 30 & 32 \\
\hline $\mathbf{0}$ & l-l & & $1-1$ & $1-1^{a}$ & $s-1^{a}$ & s-l (sf) & $s-l\left(s f^{b}\right)$ & $\mathrm{s}-\mathrm{l}(\mathrm{ax})$ \\
\hline 2 & & & $s-1^{a}$ & & & & & \\
\hline 5 & l-l & $s-1^{a}$ & $s-1^{a}$ & & $s-1(s f)$ & $\mathrm{s}-1\left(\mathrm{sf}^{\mathrm{b}}\right)$ & & kin \\
\hline 10 & s-1 (sf) & & $s-1(a x)$ & & $s-1(a x)$ & s-l (ax) & & \\
\hline 15 & & & $s-1(\operatorname{ax})$ & & $\mathrm{s}-1(\mathrm{ax})$ & kin & & \\
\hline 20 & $s-1(-)$ & & $s-1(d e)$ & & kin & & & \\
\hline 25 & un & & un & & & & & \\
\hline
\end{tabular}

l-l, liquid-liquid demixed structure; $s-1$, solid-liquid demixed structure; sf, spherulites; ax, axialites; de, dendrites; un, a solution was not obtained; and kin, occurrence of turbidity of the solution after approximately 2 days.

a $1-1^{a}, s-1^{a}$, transition from liquid-liquid to solid-liquid demixing.

${ }^{b} \mathbf{s f}^{\text {b }}$, transition sf-ax.

spherulites according to the theory of Keith and Padden as described by Khoury and Passaglia ${ }^{29}$ is shown in Figure 8. In the early stages the spherulite has a sheaflike precursor. Structures (a-d) represent the intermediate stages in the development of the spherulite. Neighboring transitional structures can impinge upon the growing units and stop further growth before they attain the spherically symmetric growth stage. The morphology of the polymer sample then is composed of units of axialites.

Spherulite-forming materials are multicomponent systems in which certain components (impurities of the crystal) are rejected preferentially by the growing crystals. The tips of growing lamellae abstract relatively pure material from the bulk and impurities accumulate between the lamellae. Spherulites are reported to be relatively open. Spherulites that are grown in an environment of polymer in a saturated solution of one liquid in another liquid (e.g., water and formic acid) will reject the low molecular weight components beside the macromolecular impurities. It can be expected that such spherulites have a 'spongy' texture. This morphology is indeed observed for the spherulites shown in Figure 4.

The size of the spherulites grown from a pure polymeric melt is generally dependent on the extent of undercooling. At high undercoolings more spherulites are nucleated per unit volume. The larger the nucleation density, the less spherulites are able to grow freely before they reach a neighboring spherulite. For our membrane system apparently the nucleation density increases with increasing polymer concentration and/or increasing water concentration in the polymer dope (Figs. 4, 5, 7).

Spherulitic structures in membranes resulting from s-l phase separation of the binary-system polypropylene in mineral oils quenched from 245 to

Table II Characteristics of Nylon 4,6 Membranes Varying as a Function of Polymer and Nonsolvent Concentrations

\begin{tabular}{|c|c|c|c|c|c|}
\hline \multicolumn{2}{|c|}{ Concentration } & \multirow{3}{*}{$\begin{array}{l}\text { Water Flux } \\
\left(\mathrm{L} / \mathrm{m}^{2} \mathrm{~h}^{-1}\right. \\
\left.\text { bar }^{-1}\right)\end{array}$} & \multirow{3}{*}{$\begin{array}{l}\text { Air Permeability } \\
\left(\mathrm{L} / \mathrm{cm}^{2} \mathrm{~min}^{-1}\right) \\
\text { at } 2 \mathrm{bar}\end{array}$} & \multirow{2}{*}{\multicolumn{2}{|c|}{ Pore Size $(\mu \mathrm{m})$}} \\
\hline & & & & & \\
\hline (wt \%) & (wt \%) & & & $(\operatorname{Max})$ & (Average) \\
\hline 20 & 5 & 15000 & 6.4 & 1.0 & 0.85 \\
\hline 20 & 10 & 4000 & 1.6 & 0.40 & 0.30 \\
\hline 25 & 0 & 13000 & 3.1 & 0.89 & 0.62 \\
\hline 25 & 5 & 1200 & 0.7 & 0.40 & 0.36 \\
\hline 25 & 10 & 800 & 0.7 & 0.20 & 0.16 \\
\hline 27.5 & 0 & 2500 & 0.74 & 0.74 & 0.45 \\
\hline 30 & 0 & 1250 & 0.33 & 0.70 & 0.28 \\
\hline 32 & 0 & 300 & 0.16 & 0.25 & 0.10 \\
\hline
\end{tabular}



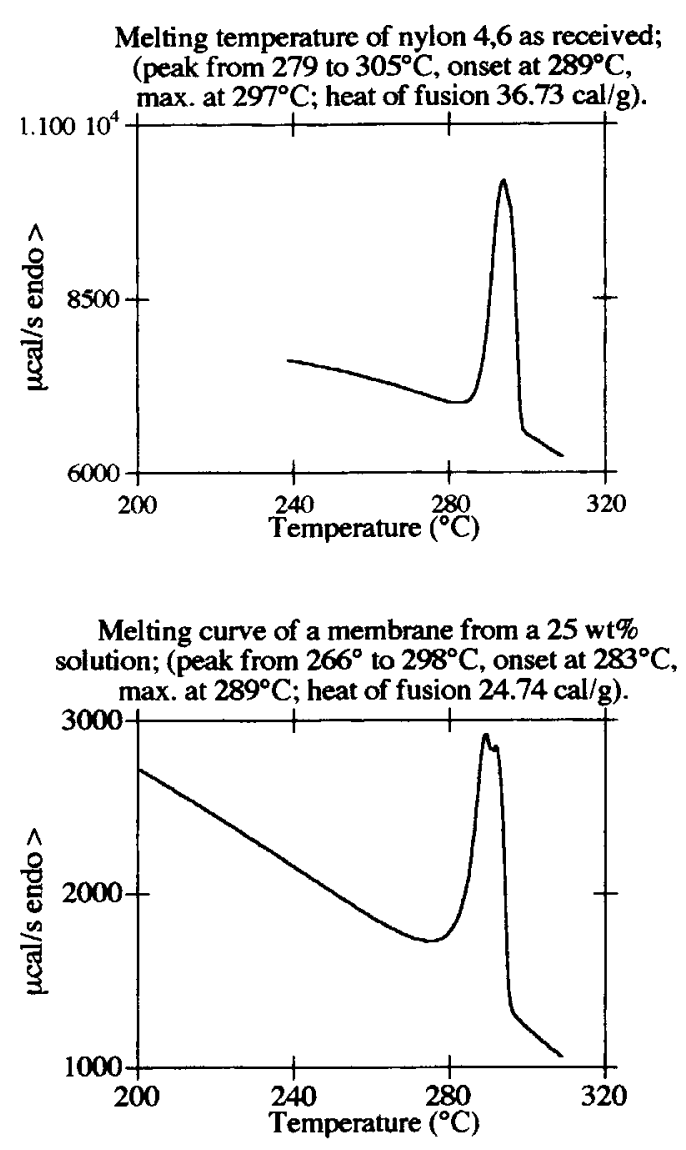

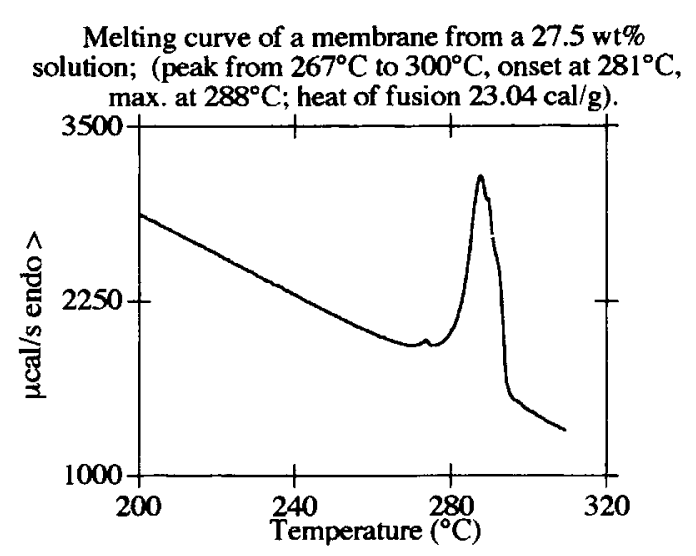

Melting curve of a membrane from a $32 \mathrm{wt} \%$ solution; (peak from 265 to $301{ }^{\circ} \mathrm{C}$, onset at $284^{\circ} \mathrm{C}$,

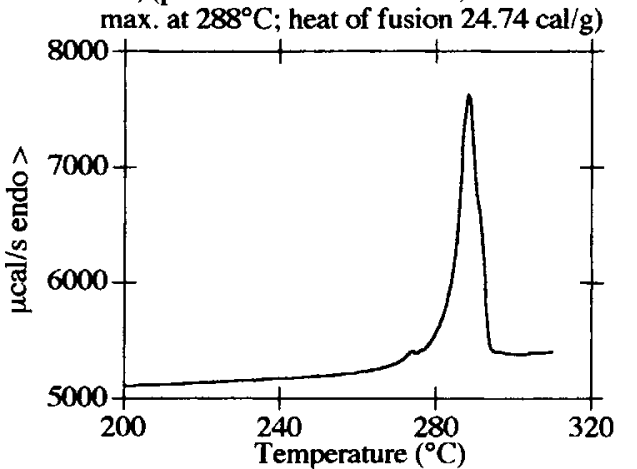

Figure 6 Differential scanning calorimetry of nylon 4,6 and of the membranes prepared from solutions with various polymer concentrations.

$43^{\circ} \mathrm{C}$ are reported by Lim et al ${ }^{10}$ The spherulite size decreased, because of an increasing nucleation density, when the cooling rate is faster and the crystallization temperature is lower or if a nucleating agent, such as adipic acid, was used.

Spherulitic growth of other polyamides from solution is reported by Keller ${ }^{30,31}$ for polyamide 6,6 and polyamide 6,10 from formic acid and cresol and by Magill ${ }^{32,33}$ for the 'odd-odd' nylons and the 'oddeven' nylons. Films were prepared by evaporation of solvent or by quenching in water.

It is expected that the membrane morphology described by Marinaccio and Knight ${ }^{20}$ for semicrystalline nylons is a result of the same type of demixing phenomena as observed by us. The authors mentioned that ". . . aggregation in solution results in film porosity since the film as cast can be thought to consist of interacting aggregation of spherical particles. The larger the sphere the larger the voids in the film. Structurally this is much like a box of tennis balls or other nonspherical geometrics fused at their point of contact. . . ." The membrane mor- phology as it is shown in the SEM photographs in the Figures 4 and 5 can be very well visualized with the previous description.

Soldatov et al. ${ }^{22}$ investigated the precipitation behavior of nylon 6 films in aqueous acetone. They discussed that with an increasing acetone content a structure conversion occurs: first the film has a cellular morphology; the use of pure acetone results in a structure composed of 'globules' with a size of 3$5 \mu \mathrm{m}$.

It can be concluded that the phenomena found for nylon 4,6 can be generalized for other aliphatic polyamides, though nylon 4,6 is a much more rapidly crystallizing nylon. Therefore the kinetical crystallization process is likely to yield a more pronounced crystalline structure for nylon 4,6 compared to the other aliphatic nylons.

\section{Solid-Liquid Demixing Versus Liquid-Liquid Demixing}

An $\mathrm{l}-\mathrm{l}$ demixing region is present for the ternary system nylon 4,6 formic acid, and water. At low 

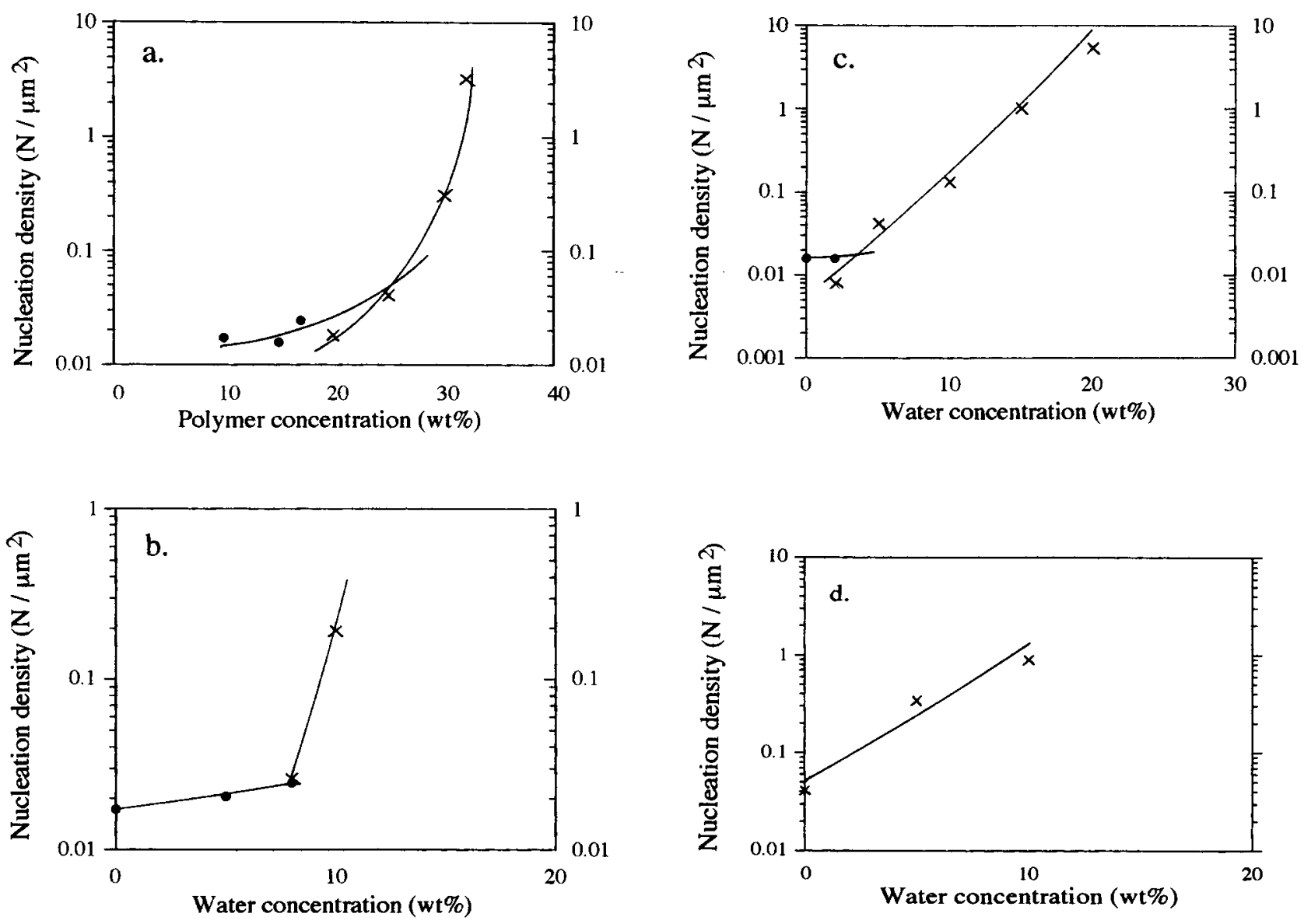

Figure 7 Nucleation density in the membranes prepared with diffusion induced phase separation as a function of the composition of the initial solution: (a) the nucleation density as a function of the initial polymer concentration; ( $b$ ) as a function of the water concentration added to a $10 \mathrm{wt} \%$ polymer solution; (c) as a function of water concentration for a $15 \mathrm{wt} \%$ polymer solution; and (d) for a $25 \mathrm{wt} \%$ polymer solution. The dots represent the nucleation density for liquid-liquid demixing, the crosses for solid-liquid demixing. The left- and right-hand ordinate give the values at different scales.

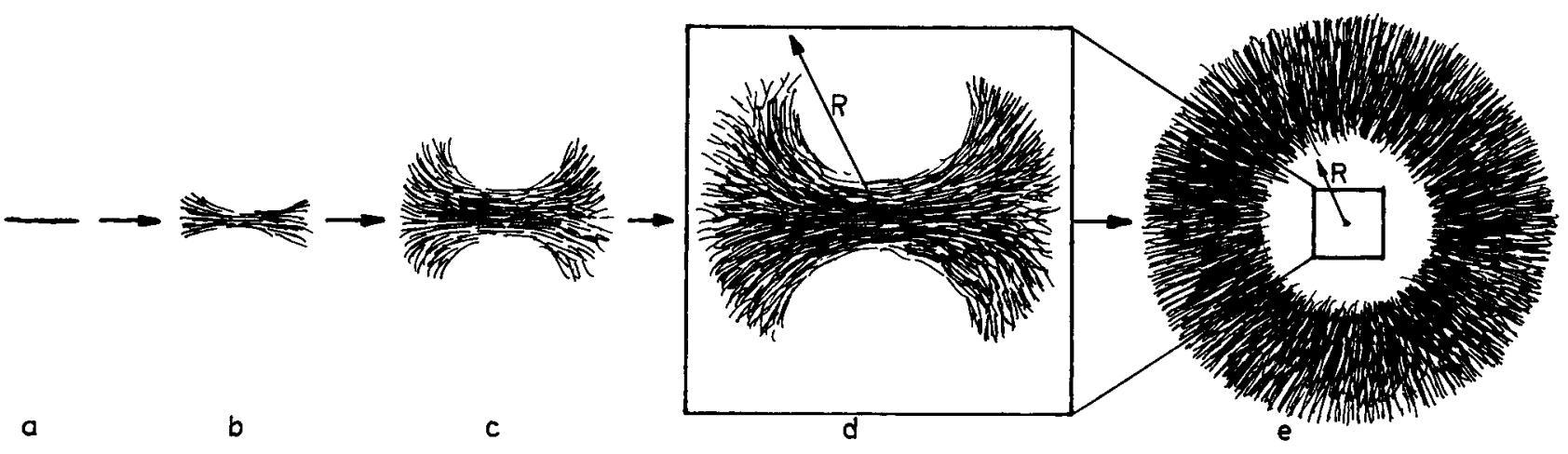

Figure 8 Schematic representation of successive stages in the development of a spherulite (structure e) from a chain-folded precursor crystal (structure a). The structures (a-d) are drawn at a larger magnification and can be found within the radius $R$ of the spherulite (structure e). 

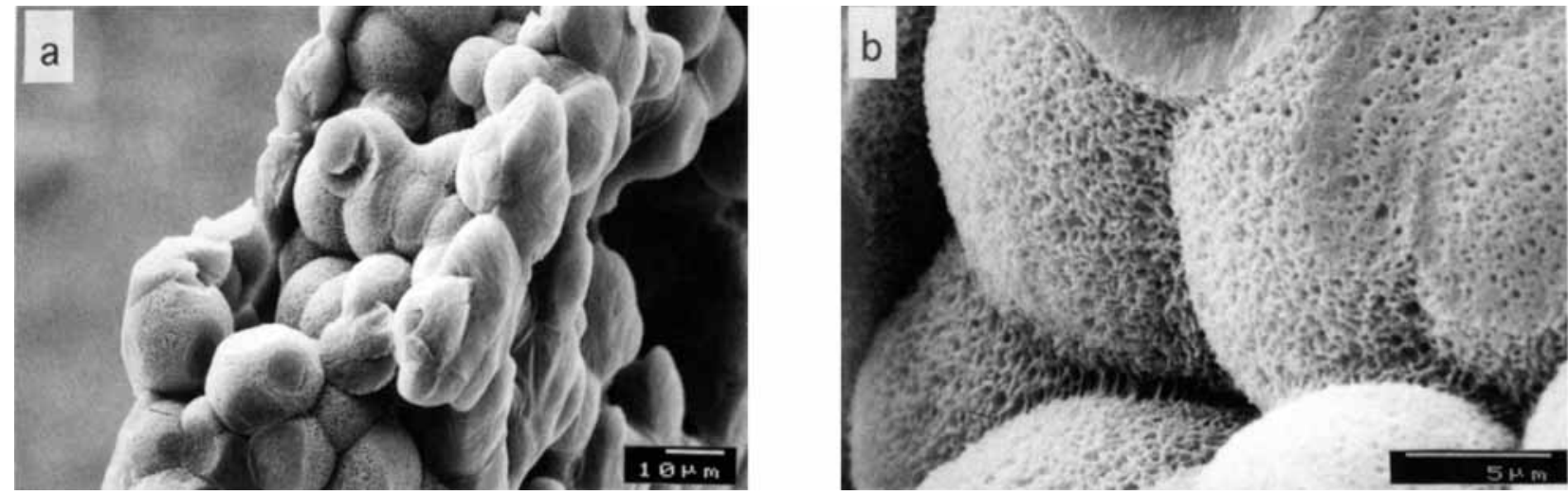

Figure 9 Membrane morphology of a 15 wt \% membrane, prepared by a diffusion induced phase separation process by a saturated vapor of water in nitrogen. The right-hand photograph is a spherulite in the membrane at a higher magnification.

polymer concentrations, for example, $15 \mathrm{wt} \%$ of polymer, the polymer solution at different positions in the film during the diffusion processes phase separate according to an $1-1$ demixing mechanism. For the cast polymer films at a polymer concentration of $15 \mathrm{wt} \%$, the polymer and the water concentration both will increase during the diffusion processes. This means that the composition passes through a region in the phase diagram, in which s-l demixing would occur, for instance when membrane formation was started with a film containing 20 or $25 \mathrm{wt} \%$ of polymer and 5 or $10 \mathrm{wt} \%$ of water (see Table I). The final membrane structure, however, differs to

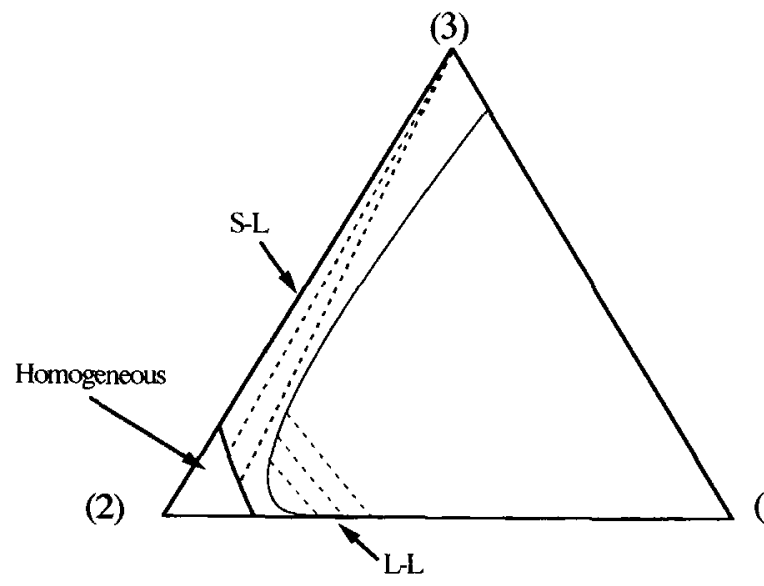

Figure 10 Schematical diagram of the position of the crystallization line and the binodal. At equilibrium conditions only the crystallization line should be drawn. For membrane formation the binodal curve is of importance here because at certain conditions crystallization may not take place as a first phase separation process because of its relatively slower demixing kinetics and/or a not high enough degree of supersaturation. a large extent. Since an s-l interface has a higher surface energy than an 1-1 interface, nucleus formation of a solid phase in a liquid is energetically much more difficult than the formation of a liquid nucleus in a liquid phase. Compositions within the $\mathrm{s}-1$ region will not nucleate easily from a homogeneous solution. Solutions with lower polymer concentrations pass through the meta-stable region ( $\mathrm{s}-$ l demixing can take place thermodynamically) toward the binodal. These solutions remain homogeneous until the $\mathrm{I}-\mathrm{I}$ binodal is crossed.

Two effects can explain s-l demixing, despite the fact that nucleation is energetically more difficult. The first one is that nuclei were already present in the solution; s-l demixing is for instance initiated by small crystallites in the solution. The process of crystal growth takes place by secondary nucleation of polymer molecules on the crystalline nucleus. For the membranes with a large nucleation density (Fig. 7) that show an axialitic structure, it is expected that a large number of small precursor crystallites are already present in the initial solution. The second possible cause is that the time available for crystallization is large enough to enable s-l demixing to take place before phase separation occurs according to an $1-1$ demixing mechanism. For the lower polymer concentrations $(<17 \mathrm{wt} \%)$ the viscosity of the solution is relatively low. Diffusion processes are fast and therefore the time that the composition in the film is in the s-l demixing gap is too short to allow a crystallization process to take place. If this is the case it is expected that crystallization will occur if the diffusion processes are slowed down. In order to verify this hypothesis, a $15 \mathrm{wt} \%$ polymer film was precipitated in a saturated water vapor atmosphere. This film exhibited l-l demixing when allowed to phase separate in a water bath (Fig. 4) . 
The morphology of the membrane precipitated from the vapor phase is shown in Figure 9. Large spherulites with a size of $20 \mu \mathrm{m}$ have grown in the membrane. Apparently the crystallization process can take place because of the slower diffusion processes of the in-diffusion of nonsolvent and the out-diffusion of solvent. The time available for a composition in the film within the s-l demixing gap is large enough to initiate the process of nucleation and growth of a ( solid) nucleus.

For this system (nylon 4,6, formic acid, and water) it can be concluded that the crystallization line is always positioned before the binodal, as is schematically shown in Figure 10. Thermodynamic crystallization is the favorable phase separation process. In some cases, however, it is possible to surpass the crystallization region, when the kinetic demixing conditions during DIPS are fast relative to the time necessary for $\mathrm{s}-1$ demixing.

Our results for the membrane formation mechanism for the nylon 4,6 membranes are in agreement with results discussed by Wijmans et al. ${ }^{16}$ and Reuvers. ${ }^{17}$ According to Reuvers crystallization is the thermodynamically most favorable state for the ternary system PPO-TCE-octanol. Demixing processes carried out with a liquid nonsolvent usually give a membrane morphology as a result of l-l phase separation. Wijman et al. ${ }^{16}$ discussed a series of experiments in which membranes were prepared by phase inversion from the vapor phase using a vapor of octanol as a nonsolvent. In contradiction to the conclusions drawn by Wymans, the SEM photos show structures that are most likely a result of a crystallization process. The rate of in-diffusion of nonsolvent and out-diffusion of solvent in a vapor phase induced demixing process is slow enough to allow crystallization to be the most important phase separation mechanism for the ternary system PPOTCE-octanol.

Solid-liquid demixing is competitive with $1-1$ demixing in a DIPS process only if small nuclei are already present in the solution (heterogeneous nucleation) or if the diffusion processes are slow enough to have enough time available for the nucleation process of a crystalline nucleus. Even while crystallization is thermodynamically the most favorable state $1-1$ demixing still can take place as a first phase separation process.

\section{CONCLUSIONS}

Crystallization ( $\mathrm{s}-\mathrm{l}$ demixing) occurs in the DIPS process for the preparation of nylon 4,6 membranes, resulting in a spherulitic or axialitic morphology of the membrane. Solid-liquid demixing takes place starting from films of solutions of nylon 4,6 in formic acid with polymer concentrations larger than 17 wt \% upon quenching in a water bath. These polyamide membranes exhibit a clear melting peak (DSC). At lower concentrations the membrane morphology is a result of $1-1$ demixing. Solid-liquid demixing is kinetically competitive with $l-l$ demixing in a DIPS process if small nuclei are already present in the starting solutions (heterogeneous nucleation) or if a relatively long time is available for the process of crystallization. The morphology resulting from $\mathrm{s}-l$ demixing is a result of spherulitic crystallization. Spherulites can grow freely if the nucleation density is small. Higher polymer concentrations and lower concentrated polymer solutions containing nonsolvent in larger concentrations give a large concentration of nuclei in the beginning. If nucleation density is large, the precursors for spherulites cannot grow until they attain the symmetric growth stage, resulting in an axialitic structure.

The authors wish to thank Stephan Eltink for the enlightening discussions and DSM The Netherlands for the funding of this project.

\section{REFERENCES}

1. R. Kesting, Synthetic Polymeric Membranes, A Structural Perspective, 2nd ed., John Wiley \& Sons, New York, 1985.

2. M. H. V. Mulder, Basic Principles of Membrane Technology, Kluwer, Dordrecht, 1991.

3. F. W. Altena and C. A. Smolders, Macromolecules, 15, 1491 ( 1982).

4. J. G. Wijmans, F. W. Altena, and C. A. Smolders, J. Polym. Sci., Polym. Phys., 22, 519 (1984).

5. A. J. Reuvers and C. A. Smolders, J. Membr. Sci., 34, 67 (1987).

6. D. Lloyd, J. Barlow, and K. Kinzer, AIChE Symposium Series, 261, 28 (1988).

7. D. Lloyd, K. Kinzer, and H. Tseng, J. Membr. Sci., 52, 239 (1990).

8. D. Lloyd, S. Kim, and K. Kinzer, J. Membr. Sci., 64, 1 (1991).

9. S. Kim and D. Lloyd, J. Membr. Sci., 64, 13 (1991).

10. G. Lim, S. Kim, Q. Ye, Y. Wang, and D. Lloyd, J. Membr. Sci., 64, 31 (1991).

11. S. Kim, G. Lim, A. Alwattari, Y. Wang, and D. Lloyd, J. Membr. Sci., 64, 41 (1991).

12. A. Alwattari and D. Lloyd, J. Membr. Sci., 64, 55 (1991).

13. F. W. Altena and C. A. Smolders, J. Polym. Sci., Poly. Symp., 69, 1 (1981).

14. P. T. van Emmerik and C. A. Smolders, J. Polym. Sci. C, 38, 73 (1972). 
15. D. M. Koenhen and C. A. Smolders, J. Polym. Sci., Polym. Phys. Ed., 15, 155 (1977).

16. J. G. Wijmans, H. J. J. Rutten, and C. A. Smolders, J. Polym. Sci., Polym. Phys. Ed., 23, 1941 (1985).

17. A. J. Reuvers, Ph.D. thesis, Appendix A, Chap. 2, University of Twente, The Netherlands, 1987.

18. W. R. Burghardt, L. Yilmaz, and A. J. McHugh, Polymer, 28, 2085 (1987).

19. A. Ladbudzinska, A. Wasiak, and A. Ziabicki, J. Polym. Sci., C16, 2835 (1967).

20. P. Marinaccio and R. Knight, U.S. Pat. $3,876,738$ (1975).

21. V. Soldatov, V. Artamov, and O. Mostovlyanskii, Proc. 29th Micro Symp. Macromolecules, Prague, 1986, p. 171.

22. P. J. Flory, Principles of Polymer Chemistry, Cornell University Press, New York, 1953.

23. F. W. Altena, J. S. Schröder, R. van de Hulst, and C. A. Smolders, J. Polym. Sci., Polym. Phys. Ed., 24, 1725 (1986).
24. D. R. Paul, J. Appl. Polym. Sci., 11, 439 (1967).

25. J. H. Hildebrand and R. L. Scott, Solubility of Nonelectrolytes, 3rd ed., Dover, Englewood Cliffs, NJ, 1964.

26. H. D. W. Roesink, Ph.D. thesis, University of Twente, The Netherlands, 1989.

27. D. Pall, U.S. Pat. 4,340,479 (1982).

28. J. F. Ditter, W.O. Pat. 86/07544 (1986).

29. F. Khoury and E. Passaglia, in The Morphology of Crystalline Synthetic Polymers, Treatise on Solid State Chemistry, Vol. 3, Chap. 6, N. B. Hannay, Ed. Plenum Press, New York, 1976.

30. A. Keller, J. Polym. Sci., 17, 447 (1955).

31. A. Keller, J. Polym. Sci., 26, 361 (1959).

32. J. Magill, J. Polym. Sci. A, 9, 817 (1971).

33. J. Magill, J. Polym. Sci. A, 3, 1193 (1965).

Received July 16, 1992

Accepted December 14, 1992 